\title{
Combining Super Resolution Algorithm (Gaussian Denoising and Kernel Blurring) and Compare with Camera Super Resolution
}

\author{
Muhamad Ghofur ${ }^{* *}$, Tjong Wan Sen ${ }^{2}$ \\ ${ }^{1,2}$ Information Technology, Faculty of Computing, President University \\ Email: ${ }^{1}$ muhamad.ghofur@student.president.ac.id, ${ }^{2}$ wansen@president.ac.id
}

\begin{abstract}
This problem addresses the problem of low-resolution image (noisy) that will proof later by PSNR number. The best way to improve this low-resolution problem is by utilizing Super Resolution (SR) algorithm methodology. SR algorithm methodology refers to the process of obtaining higher-resolution images from several lower-resolution ones, that is resolution enhancement. The quality improvement is caused by fractional-pixel displacements between images. SR allows overcoming the limitations of the imaging system (resolving limit of the sensors) without the need for additional hardware. This research aims to find the best SR algorithm in form of stand-alone algorithm or combine algorithm by comparing with the latest SR algorithm (Camera SR) from the previous research made by Chang Chen et al in 2019. Furthermore, we confidence this research will become the future guideline for anyone who want to improve the limitation of their low-resolution camera or vision sensor by implementing those SR algorithms.
\end{abstract}

Keywords - Camera, Image, Resolution.

\section{INTRODUCTION}

Image resolution describes the amount of information contained by images. Lower resolution less would be the amount of information, higher resolution more would be amount of information in images. Resolution of a digital image can be classified in many ways: pixel resolution, spatial resolution, spectral resolution, temporal resolution, and radiometric resolution. In this resaerch concentration is given mainly in spatial resolution. Spatial resolution: A digital image is made from small picture elements called pixels. Spatial resolution refers to the pixel density in an image and measures in pixels per unit area.

The spatial resolution of an image is limited by the image sensors or the image acquisition devices. The modern image acquisition devices are using charge-coupled device (CCD) or complementary metal oxide semiconductor (CMOS) as active pixel sensor. These sensors are arranged in two dimensional arrays to capture twodimensional image signals. The number of sensors per unit area or the sensor size determines the number of pixels in image. One way to increase the resolution of the imaging device is to increase the sensor density by reducing the size of sensors. When the size of sensors is reduced beyond a limit it causes shot noise in the captured images as reducing the size of sensor also reduces the amount of light incident on it. Increment in the number of sensors in imaging device/system also increases the hardware cost. Therefore, there is limitation with the hardware that restricts the spatial resolution of the image.

While spatial resolution is limited by sensor size, the image details (high frequency bands) are also limited by the optics due to lens blurs (associated with the sensor point spread function (PSF)), lens aberration effects, aperture diffractions and optical blurring due to motion. Constructing imaging chips and optical components to capture very high-resolution images is prohibitively expensive and not practical in most real applications, e.g., widely use surveillance cameras and cell phone built-in cameras. In some other scenarios such as satellite imagery, it is difficult to use high resolution sensors due to physical constraints.

Another way to address this problem is to accept the image degradations and use image processing to post process the captured images, to trade of computational cost with the hardware cost. These techniques are specifically referred as super resolution (SR) reconstruction.

Single image super-resolution (SR) is a typical inverse problem in computer vision. Generally, $S R$ methods assume bicubic or Gaussian down sampling as the degradation model [1]. Based on this assumption, continuous progress has been achieved to restore a better high-resolution (HR) image from its low-resolution (LR) version, in terms of reconstruction accuracy [2],[3],[4],[5],[6],[7],[8],[9],[10],[11] or perceptual quality [12],[13],[14],[15],[16],[17],[18] However, these synthetic degradation models may deviate from the ones in realistic imaging systems, which results in a significant deterioration on the SR performance [19]. To better simulate the challenging real-world conditions, additional factors including noise, motion blur, and compression artifacts are integrated to characterize the LR images in either a synthetic [20] or a data-driven [21] manner.

\section{RESEARCH METHODOLOGY}

All methods that will be run in this research can be described in below process flow diagram. 


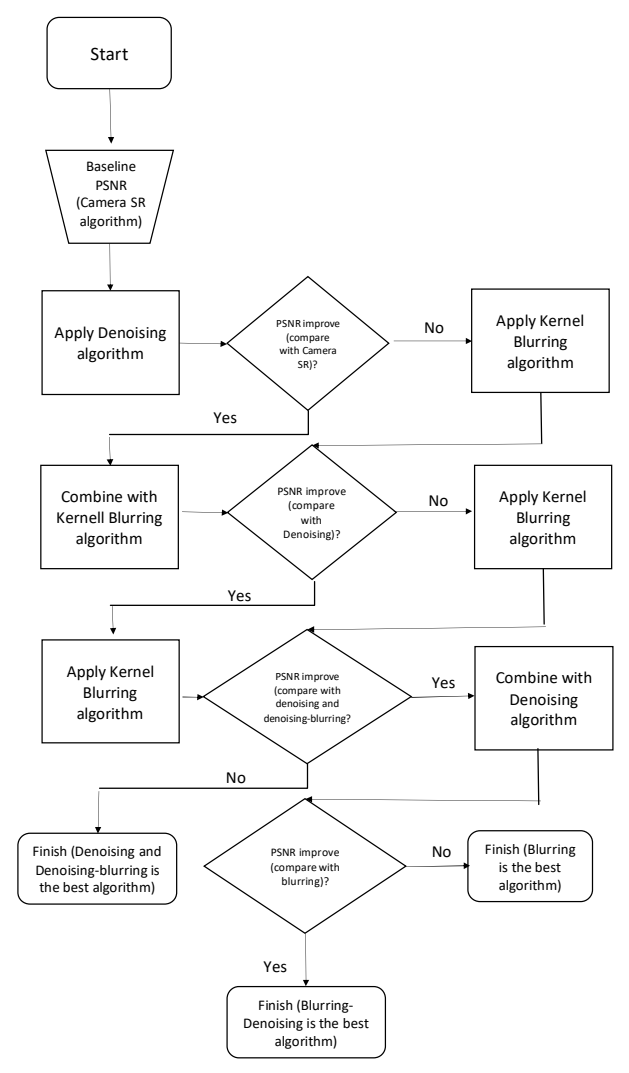

Figure. 1 Research Method.

All methods that will be run step by step as described in above process flow are explained below:

1. Get baseline picture sample.

In this method, we will use pictures from City100 data set. This is a set of pictures that were taken from some postcards and did it indoor. After that run Camera SR to these data set.

2. Apply SR Algorithm

After got the baseline data, then the next method that we will do is applying all those algorithms (stand-alone and combination). SR Algorithm that will applied in this research are Gaussian denoising, Kernel blurring, Denoising-Blurring and Blurring-Denoising.

After that, every time we finished to apply each algorithm, we will compare their PSNR score with Camera SR PSNR score to get which one is the best SR algorithm if we refer with their PSNR score.

\section{RESULTS AND DISCUSSION}

We will start with showing some pictures from City100 data set and our CCD picture dataset. This will become base line picture data. Then after that it will proceed with Super Resolution algorithm in sequence and vice versa. We have set a requirement in this research method, that if there is any algorithm which does not give improvement in the picture data set then we will continue with this algorithm.

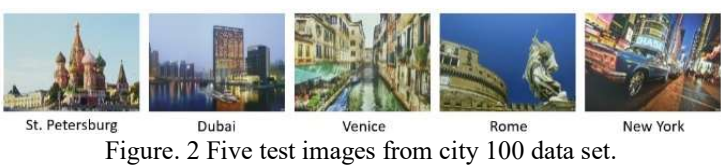

\section{A. Kernel Blurring}

A kernel is a (usually) small matrix of numbers that is used in image convolutions, while convolution itself is a general-purpose filter effect for images. Convolution filtering is usually used to modify the spatial frequency characteristics of an image. They did it by applying a matrix to an image and a mathematical operation comprised of integers. The mathematical operation will work by determining the value of a central pixel by adding the weighted values of all its neighbors together. Then the output is a new modified filtered image. Different sized kernels containing different patterns of numbers produce different results under convolution. The image blurring process is commonly modeled as the convolution of a clear image with a shift-invariant kernel plus noise, i.e.,

$$
f=k * g+n
$$

where $*$ denotes the discrete convolution operator, $g$ denotes the clear image, $g$ denotes the available blurry observation, $k$ denotes the blur kernel, and $\mathrm{n}$ denotes the image noise. The size of a kernel is arbitrary but $3 \mathrm{X} 3$ is often used.

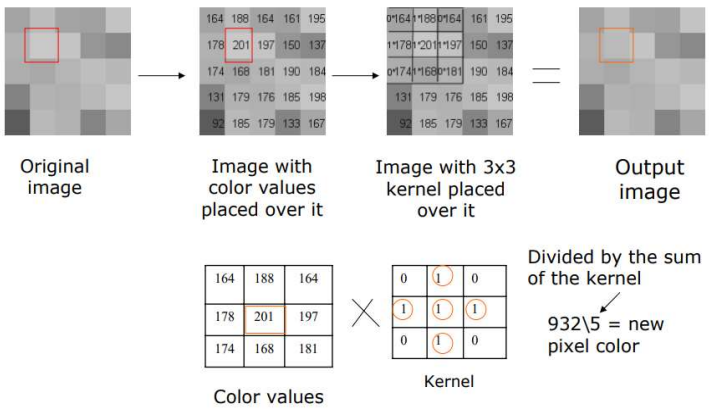

Figure. 3 Kernel matrix.

Based on our research, to get higher PSNR we must use a square kernel matrix $(3 \times 3,5 \times 5$, etc.). When we did not use a square kernel matrix, the color matrix distribution will not even and created some pictures spot greyish or darker. Based on our research, the best kernel matrix square that get the highest PSNR is $5 \mathrm{X} 5$ matrix. 


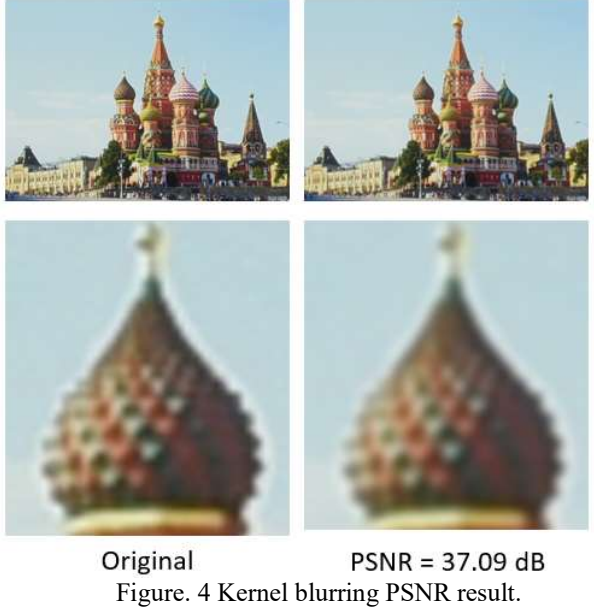

B. Gaussian Denoising

The probability density function $\mathrm{p}$ of a Gaussian random $\mathrm{z}$ variable is given by:

$$
p G(z)=\frac{1}{\sigma \sqrt{2 \pi}} e^{-\frac{(x-\mu)^{2}}{2 \sigma^{2}}}
$$

where $\mathrm{z}$ represents the grey level, $\mu$ the mean grey value and $\sigma$ its standard deviation. In digital image processing Gaussian noise can be reduced using a spatial filter, though when smoothing an image, an undesirable outcome may result in the blurring of fine-scaled image edges and details because they also correspond to blocked high frequencies. The different with kernel blurring is in denoising the result will be based on how big the noise filter. The bigger noise filter, the bigger high frequency cannot be passed. Based on our research the noise filter $=$ 25 gave the highest PSNR result.

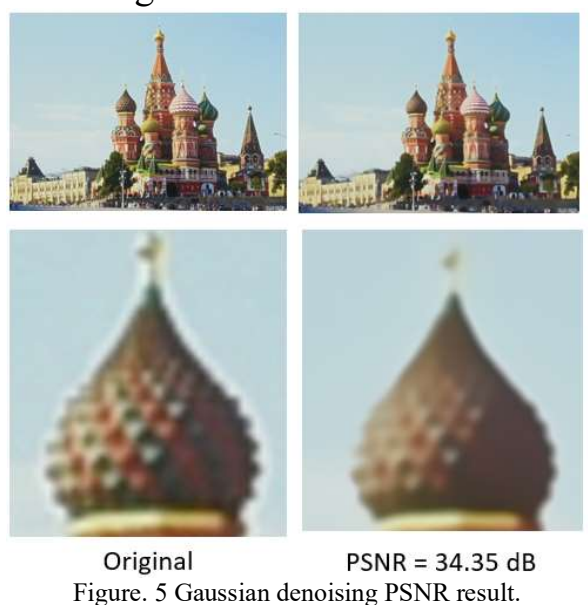

C. Blurring and Denoising

Here are the PSNR results after we combine Blurring and Denoising.

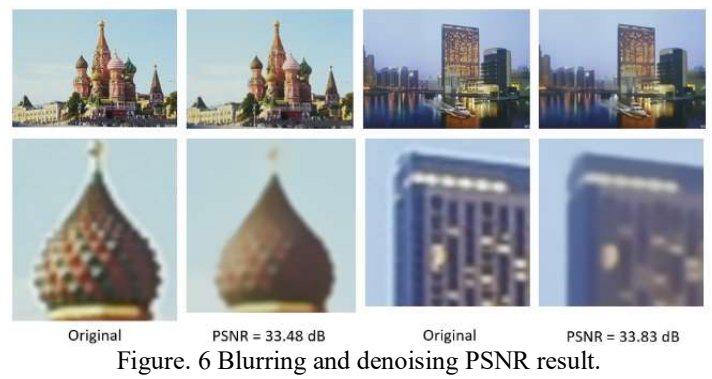

D. Denoising and Blurring

Here are the PSNR results after we combine Denoising and Blurring.

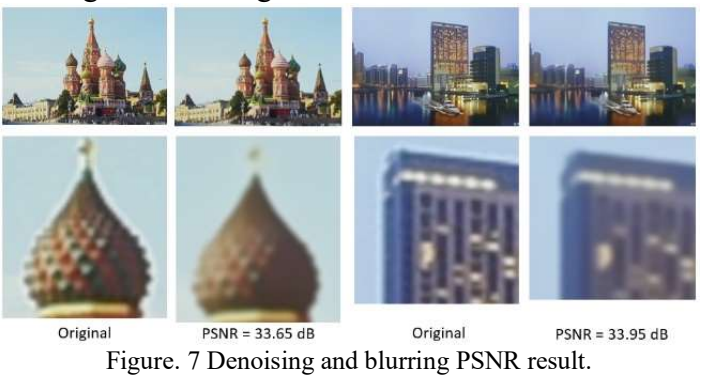

E. Result Summary

After we include with the result from previous research by using Camera SR methodology, here is the PSNR result summary from each algorithm methodology and their combination.

Table 1. Research Summary

\begin{tabular}{l|l|l|l|l|l|}
\hline Picture & Blurring & Denoising & $\begin{array}{l}\text { Blurring } \\
- \\
\text { Denoising }\end{array}$ & $\begin{array}{l}\text { Denoising } \\
- \\
\text { Blurring }\end{array}$ & $\begin{array}{l}\text { Camera } \\
\text { SR }\end{array}$ \\
\hline $\begin{array}{l}\text { St. } \\
\text { Petersburg. }\end{array}$ & 37.09 & 34.35 & 33.48 & 33.65 & 31.00 \\
\hline Dubai & 38.77 & 34.50 & 33.83 & 33.95 & 31.94 \\
\hline Venice & 35.00 & 31.46 & 30.52 & 30.68 & 28.19 \\
\hline Rome & 37.85 & 34.42 & 33.46 & 33.64 & 33.04 \\
\hline New York & 34.96 & 32.64 & 31.50 & 31.68 & 27.14 \\
\hline Average & 36.73 & 33.47 & 32.56 & 32.72 & 30.26 \\
\hline
\end{tabular}

By looking at the PSNR (dB) result in above table we can see that by our research, with picture characteristics in City100 data set, by only utilize one algorithm methodology that is Kernel Blurring actually we can get the best picture quality. How it can be happened? Because theoretically, a kernel blurring is a (usually) small matrix of numbers that is used in image convolutions, while convolution itself is a general-purpose filter effect for images. By using Kernel, a single matrix color can distribute some parts of their color to their neighborhood matrix. It is not just like a denoising algorithm when they want to be smoothing the noise using their spatial filter, an undesirable outcome may result in the blurring of finescaled image edges and details because they also correspond to blocked high frequencies (due to their power of noise filter). 


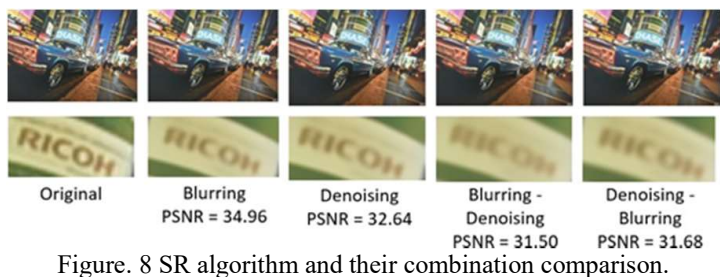

However, for all SR algorithm and combination if we look at the pictures result each of them, we can see that some effect may happened when we zoom it in a specific point. We can get a blurred fine scaled image even though in overall picture result we can see a better-quality picture. This is the important thing we may consider when we want to implement these Super Resolution algorithm for our image processing. Because in some cases when you already have a clean (free or less noise) picture, you may not need these SR algorithms anymore. Or perhaps you need another SR algorithm like Interpolation for the example if you want to play with bigger pixel picture.

\section{CONCLUSION}

In this research we try to analyze the benefit of some Super Algorithm methodology and their combination to find solution for pictures with noise characteristics. We also want to compare these solutions with Camera SR methodology that previously has been proposed by Chen et al. We use the same data set that is City100 that been developed by Chen et al in their paper in 2019. Based on City100, we analyze the advantage and disadvantage of some SR algorithm including Camera SR methodology and validate that actually one of the algorithms that is Kernel Blurring is the best algorithm of other existing SR methods with average PSNR $36.73 \mathrm{~dB}$ and a practical solution so far to boost the performance of noisy pictures. Perhaps Chen et al. did not consider "Kernel Blurring" algorithm in their paper as we did not see this algorithm was discussed and analyzed in their paper. Despite the validating result, there are still some updated methodology and picture aspects that we have not touched in our research. Like for example how to increase pixel quantity, because increasing pixel quantity is also part of Super Resolution works. While in the updated methodology, perhaps we also can utilize the use of Deep Learning methodology to predict how Neural Network in a computer can do an automatic convolutional filter. The above example will be considered as our future works.

\section{REFERENCES}

[1] K. Zhang, W. Zuo, and L. Zhang, "Learning a Single Convolutional Super-Resolution Network for Multiple Degradations," 2018, doi: 10.1109/CVPR.2018.00344.

[2] M. Haris, G. Shakhnarovich, and N. Ukita, "Deep Back-Projection Networks for Super-Resolution,"
2018, doi: 10.1109/CVPR.2018.00179.

J. Kim, J. K. Lee, and K. M. Lee, "Accurate image super-resolution using very deep convolutional networks," in Proceedings of the IEEE Computer Society Conference on Computer Vision and Pattern Recognition, 2016, vol. 2016December, doi: 10.1109/CVPR.2016.182.

W. S. Lai, J. Bin Huang, N. Ahuja, and M. H. Yang, "Deep laplacian pyramid networks for fast and accurate super-resolution," in Proceedings 30th IEEE Conference on Computer Vision and Pattern Recognition, CVPR 2017, 2017, vol. 2017-January, doi: 10.1109/CVPR.2017.618.

B. Lim, S. Son, H. Kim, S. Nah, and K. M. Lee, "Enhanced Deep Residual Networks for Single Image Super-Resolution," in IEEE Computer Society Conference on Computer Vision and Pattern Recognition Workshops, 2017, vol. 2017July, doi: 10.1109/CVPRW.2017.151.

[6] A. Shocher, N. Cohen, and M. Irani, "Zero-Shot Super-Resolution Using Deep Internal Learning," 2018, doi: 10.1109/CVPR.2018.00329.

[7] Y. Tai, J. Yang, and X. Liu, "Image superresolution via deep recursive residual network," in Proceedings - 30th IEEE Conference on Computer Vision and Pattern Recognition, CVPR 2017, 2017, vol. 2017-January, doi: 10.1109/CVPR.2017.298.

[8] T. Tong, G. Li, X. Liu, and Q. Gao, "Image Super-Resolution Using Dense Skip Connections," in Proceedings of the IEEE International Conference on Computer Vision, 2017, vol. 2017-October, doi: 10.1109/ICCV.2017.514

[9] Z. Xiong, X. Sun, and F. Wu, "Robust web image/video super-resolution," IEEE Trans. Image Process., vol. 19, no. 8, 2010, doi: 10.1109/TIP.2010.2045707.

[10] Y. Zhang, K. Li, K. Li, L. Wang, B. Zhong, and Y. Fu, "Image super-resolution using very deep residual channel attention networks," in Lecture 
Notes in Computer Science (including subseries Lecture Notes in Artificial Intelligence and Lecture Notes in Bioinformatics), 2018, vol. 11211 LNCS, doi: 10.1007/978-3-030-01234$2 \_18$.

[11] Y. Zhang, Y. Tian, Y. Kong, B. Zhong, and Y. Fu, "Residual Dense Network for Image SuperResolution," 2018, doi: 10.1109/CVPR.2018.00262.

[12] A. Bulat and G. Tzimiropoulos, "Super-FAN: Integrated Facial Landmark Localization and Super-Resolution of Real-World Low Resolution Faces in Arbitrary Poses with GANs," 2018, doi: 10.1109/CVPR.2018.00019.

[13] Y. Blau, R. Mechrez, R. Timofte, T. Michaeli, and L. Zelnik-Manor, "The 2018 PIRM challenge on perceptual image super-resolution," in Lecture Notes in Computer Science (including subseries Lecture Notes in Artificial Intelligence and Lecture Notes in Bioinformatics), 2019, vol. 11133 LNCS, doi: 10.1007/978-3-030-110215_21.

[14] X. Deng, "Enhancing Image Quality via Style Transfer for Single Image Super-Resolution," IEEE Signal Process. Lett., vol. 25, no. 4, 2018, doi: 10.1109/LSP.2018.2805809.

[15] J. Johnson, A. Alahi, and L. Fei-Fei, "Perceptual losses for real-time style transfer and superresolution," in Lecture Notes in Computer Science (including subseries Lecture Notes in Artificial Intelligence and Lecture Notes in Bioinformatics), 2016, vol. 9906 LNCS, doi: 10.1007/978-3-31946475-6_43.

[16] C. Ledig et al., "Photo-realistic single image super-resolution using a generative adversarial network," in Proceedings - 30th IEEE Conference on Computer Vision and Pattern Recognition, CVPR 2017, 2017, vol. 2017-January, doi: 10.1109/CVPR.2017.19.

[17] M. S. M. Sajjadi, B. Scholkopf, and M. Hirsch, "EnhanceNet: Single Image Super-Resolution
Through Automated Texture Synthesis," in Proceedings of the IEEE International Conference on Computer Vision, 2017, vol. 2017-October, doi: 10.1109/ICCV.2017.481.

[18] X. Wang, K. Yu, C. Dong, and C. Change Loy, "Recovering Realistic Texture in Image SuperResolution by Deep Spatial Feature Transform," 2018, doi: 10.1109/CVPR.2018.00070.

[19] T. Michaeli and M. Irani, "Nonparametric blind super-resolution," 2013, doi: 10.1109/ICCV.2013.121

[20] R. Timofte, S. Gu, L. Van Gool, L. Zhang, and M. H. Yang, "NTIRE 2018 challenge on single image super-resolution: Methods and results," in IEEE Computer Society Conference on Computer Vision and Pattern Recognition Workshops, 2018, vol. 2018-June, doi: 10.1109/CVPRW.2018.00130.

[21] A. Bulat, J. Yang, and G. Tzimiropoulos, "To learn image super-resolution, use a GAN to learn how to do image degradation first," in Lecture Notes in Computer Science (including subseries Lecture Notes in Artificial Intelligence and Lecture Notes in Bioinformatics), 2018, vol. 11210 LNCS, doi: 10.1007/978-3-030-01231$1 \_12$.

JISA (Jurnal Informatika dan Sains) (e-ISSN: 2614-8404) is published by Program Studi Teknik Informatika, Universitas Trilogi under Creative Commons Attribution-ShareAlike 4.0 International License. 